\title{
Prevalence of mitochondrial DNA mutations in childhood/congenital onset non-syndromal sensorineural hearing impairment
}

\author{
T P Hutchin, K R Thompson, M Parker, V Newton, M Bitner-Glindzicz, R F Mueller
}

Molecular Medicine Unit, Clinical Sciences Building, St James's University Hospital, Beckett Street, Leeds LS9 7TF, UK

T P Hutchin K R Thompson

Department of Clinical Genetics, Leicester Royal Infirmary, Leicester, UK M Parker

Centre for Audiology, Education of the Deaf and Speech Pathology, University of

Manchester,

Manchester, UK

V Newton

Institute of Child Health, University College London Medical School, London, UK

M Bitner-Glindzicz

Department of Clinical Genetics, St James's University Hospital, Leeds, UK

R F Mueller

Correspondence to:

Dr Hutchin,

t.p.hutchin@leeds.ac.uk

Revised version received 25 January 2001

Accepted for publication

30 January 2001

\begin{abstract}
Genetic factors are the major causes of childhood hearing impairment. Whereas autosomal recessive mutations account for the majority of prelingual nonsyndromic sensorineural hearing impairment (NSSHI), the relative contribution of mitochondrial DNA (mtDNA) mutations to childhood onset NSSHI has not been established.

We screened 202 subjects with congenital/ childhood onset NSSHI, consisting of 110 sporadic cases, 75 sib pairs, and 17 families with affected subjects in more than one generation, in order to determine the prevalence of mtDNA mutations associated with NSSHI.

mtDNA mutations were found in three of 10 families $(30 \%)$ in whom the affected members were related through the maternal lineage. One sporadic case $(0.9 \%)$ was also found to have a known mtDNA mutation but none was found in the sib pairs. Although the prevalence of mtDNA mutations was low in the group as a whole $(2 \%)$, we suggest that screening should be considered in cases of childhood hearing impairment when it is progressive and particularly in families where transmission is compatible with maternal inheritance.

(F Med Genet 2001;38:229-231)
\end{abstract}

Keywords: mitochondrial DNA; point mutation; hearing impairment

Hereditary hearing impairment affects approximately 1 in 2000 children. ${ }^{1}$ The majority of cases are non-syndromal and sensorineural owing to a defect in the inner ear. Nonsyndromal sensorineural hearing impairment (NSSHI) can be caused by defects in nuclear encoded genes or those of the mitochondrial DNA (mtDNA) ${ }^{23}$ and hence may be inherited as an autosomal recessive, autosomal dominant, X linked, or maternally transmitted trait. Epidemiological studies have estimated that prelingual hearing impairment is inherited in a predominantly autosomal recessive manner $(\sim 80-85 \%){ }^{1}$ Of the remaining cases, about $15 \%$ are thought to be inherited as an autosomal dominant trait, with $\mathrm{X}$ linked and mitochondrial inheritance constituting around $1 \%$. However, a more precise estimate of the relative contribution of mtDNA mutations to congenital/childhood onset hearing loss is not available. Since these early estimates often did not consider non-Mendelian inheritance, these figures may be an underestimate. We therefore screened a random sample of congenital/ childhood onset NSSHI subjects for known mtDNA mutations (A1555G, A3243G, A7445G, T7510C, T7511C, T7512C, and 7472 ins C) $)^{2-4}$ in an attempt to provide a more accurate estimate of the contribution of these mutations to such hearing impairment.

\section{Subjects and methods}

A total of 202 subjects with NSSHI were analysed. They all had sensorineural hearing impairment which was of congenital/early childhood onset. One subject had a history of aminoglycoside antibiotic exposure.

There was no family history of hearing impairment in 110 of the subjects (that is, sporadic) and 75 had one or more affected sibs but no other family history of hearing impairment. The remaining 17 subjects all had a family history of hearing impairment in subjects from at least two generations, with 10 families showing a pattern of transmission only through the maternal lineage.

Following informed consent, DNA from whole blood was screened for mtDNA mutations using restriction fragment polymorphism analysis (RFLP) for each of the following mutations associated with non-syndromal hearing impairment: A1555G, A3243G, A7445G, 7472insC, T7510C, T7511C, and T7512C.

To detect the A1555G and A3243G mutations, mtDNA between base pairs 1011 and 3376 was amplified by PCR and digested with BsmAI (A1555G) or Bsp120I (A3243G). The PCR fragment normally contains one of each site with the A1555G mutation abolishing the BsmAI site and A3243G creating another Bsp120I site.

Mutations A7445G, T7510C, and T7511C were detected by amplification of base pairs 7392 to 7608 and digestion with the appropriate restriction enzyme. A7445G abolishes an XbaI site while T7510C and T7511C create HinfI and MboII sites, respectively. The normal PCR fragment contained one of each site to check for complete digestion.

Since the 7472insC does not create or abolish a restriction enzyme site, nucleotides 7446 to 7541 were amplified by PCR using a forward primer with a mismatch to create an $X \mathrm{cmI}$ site when the 7472 ins C mutation is present. Similarly, a mismatch primer was used to create a DdeI site when the T7512C mutation was present. 
Table 1 mtDNA mutations detected in cases of congenital/childhood onset NSSHI

\begin{tabular}{|c|c|c|c|c|c|}
\hline \multirow[b]{2}{*}{ Gene } & \multirow[b]{2}{*}{ Mutation } & \multicolumn{2}{|l|}{ Familial } & \multirow[b]{2}{*}{$\begin{array}{l}\text { Sporadic } \\
(n=110)\end{array}$} & \\
\hline & & Sibs $(n=75)$ & $\begin{array}{l}>1 \text { generation } \\
\text { affected }^{\star}(n=17)\end{array}$ & & \\
\hline 12SrRNA & A1555G & $0 / 75$ & $0 / 17$ & $0 / 110$ & \\
\hline \multirow[t]{5}{*}{$\mathrm{tRNA}^{\mathrm{Ser}(\mathrm{UCN})}$} & A7445G & $0 / 75$ & $1 / 17$ & $0 / 110$ & \\
\hline & 7472insC & $0 / 75$ & $0 / 17$ & $1 / 110$ & \\
\hline & $\mathrm{T} 7510 \mathrm{C}$ & $0 / 75$ & $1 / 17$ & $0 / 110$ & \\
\hline & $\mathrm{T} 7511 \mathrm{C}$ & $0 / 75$ & $0 / 17$ & $0 / 110$ & \\
\hline & T7512C & $0 / 75$ & $0 / 17$ & $0 / 110$ & \\
\hline tRNA $^{\text {Leu(UUR) }}$ & A3243G & $0 / 75$ & $1 / 17$ & $0 / 110$ & \\
\hline Total & & $0 / 75(0 \%)$ & $3 / 17(17.6 \%)$ & $1 / 110(0.9 \%)$ & $4 / 202(2 \%)$ \\
\hline
\end{tabular}

^In 10 families, hearing impairment was transmitted only through the maternal lineage.

All restriction digests were carried out following the manufacturer's instructions (New England Biolabs and Fermentas) and separated on $2 \%$ agarose gels, stained with ethidium bromide or silver staining on a $12 \%$ polyacrylamide gel. The level of heteroplasmy was estimated by densitometry scanning of the agarose gels. The presence of each mutation was confirmed by fluorescent sequencing on an ABI sequencer 377 .

\section{Results}

The findings from this study are summarised in table 1 .

Three subjects with a family history of hearing impairment were found to carry mtDNA mutations, A7445G, T7510C, and A3243G. In all cases the hearing impairment was inherited only through the maternal lineage, that is, $30 \%$ of such families. Typical of mitochondrial hearing impairment, the age of onset and severity of hearing impairment varied widely within each family, though it was always sensorineural and progressive.

The A3243G mutation was heteroplasmic, present at a level of approximately $10 \%$ in the blood of the proband, who had moderate, progressive hearing loss first noticed at about the age of 5 . His mother and aunt were similarly affected from early teens as were several other family members. No other clinical features were apparent in any family members.

The proband with the $\mathrm{T} 7510 \mathrm{C}$ mutation had a profound hearing impairment from 15 months of age. His sister had a severe, progressive hearing impairment from the age of 5 years, whereas their mother had only a slight hearing loss. In all three subjects, the mutation was heteroplasmic and present at similar levels (about 95\%).

The family with the A7445G mutation reinforced an important principle in identifying maternally inherited hearing impairment (fig 1). Initially, the inheritance pattern was thought to be autosomal dominant because of an instance of male to male transmission. However, confirmation of the cause of hearing impairment by review of the medical notes of the affected family members showed that the child's hearing impairment was the result of glue ear. The pattern of inherited hearing impairment over three generations in the other affected family members was consistent with maternal inheritance. The A7445G mutation was homoplasmic in all family members

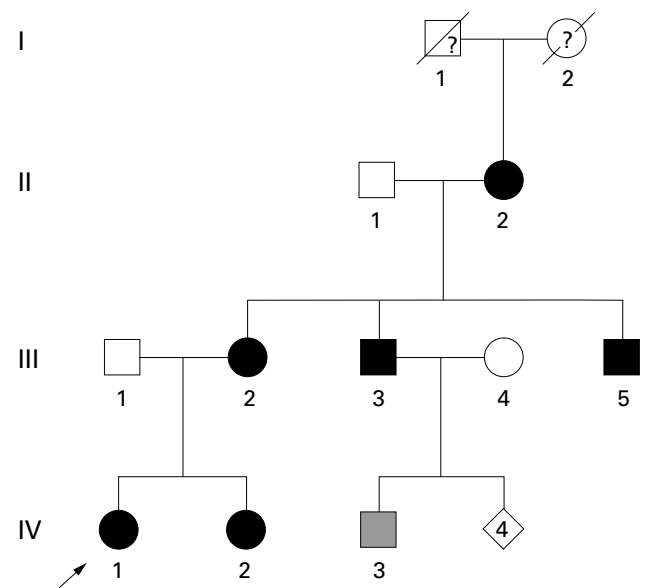

Figure 1 Family with the A7445G mutation. Solid symbols represent subjects with NSSHI, striped symbol represents NSSHI resulting from otitis media.

studied, causing a moderate to severe, progressive, asymmetrical hearing loss with an onset in early childhood. There was no evidence of other features such as palmoplantar keratoderma, which has been reported in other families with the A7445G mutation. ${ }^{2}{ }^{3}$ Further details of the families with the A7445G and T7510C mutations are described elsewhere. ${ }^{56}$

In the sporadic cases, one subject $(0.9 \%)$ was found to have the 7472insC mutation. The mutation was heteroplasmic at a level of approximately $90 \%$. Progressive hearing impairment in this child was first noticed in early childhood, but no neurological signs were present as have been reported in some subjects with this mutation. ${ }^{34}$ Although other family members also had the 7472insC mutation, their hearing was normal.

None of the known mtDNA mutations associated with NSSHI were found in the 75 sib pairs. Nor was the A1555G mutation found, although only one subject was known to have received aminoglycoside antibiotics.

\section{Discussion}

Previous estimates of the contribution of mtDNA mutations to congenital/childhood hearing impairment have largely been based on the mode of transmission of the hearing impairment over several generations. Maternal inheritance of hearing impairment can sometimes be difficult to identify, especially in small families, where there is reduced penetrance, or, as we found in one family described here, by the presence of a phenocopy. Since many early surveys often did not consider non-Mendelian inheritance, estimates of the contribution of mtDNA mutations to childhood hearing impairment may have been underestimated, as our figure of $2 \%$ suggests. Of the mutations found in this study, A1555G, A7445G, and 7472ins $C$ have previously been shown to cause hearing loss before the age of 4 in some subjects, although the age of onset often varies widely, even within a family as we found here. ${ }^{4-11}$

Two previous studies from Finland and Japan on subjects with hearing impairment of all ages found mtDNA mutations in $6.8 \%$ and 
$5.7 \%$ of NSSHI, respectively, ${ }^{72}$ although the former group were selected for those with matrilinear hearing impairment. Given that mtDNA mutations are more commonly associated with later onset hearing impairment and differences in patient selection, our finding of $2 \%$ in childhood cases is consistent with these studies. Furthermore, there are likely to be population differences, neither of the previous surveys finding the A7445G or 7472 insC mutations in the Japanese or Finnish populations.

One slightly unexpected finding in our survey was the absence of the A1555G mutation. The A $1555 \mathrm{G}$ mutation appears to be one of the most common genetic causes of hearing loss in China and $\operatorname{Japan}^{812}$ and has been reported in a number of families from Spain and Finland. ${ }^{7}{ }^{9}$ However, the absence of the mutation in our subjects is perhaps not surprising given that, except in the presence of aminoglycosides, the mutation usually gives rise to hearing impairment with an onset later in life. The A1555G mutation may be present in the population, but since few children in the UK receive aminoglycosides their hearing is unlikely to be affected at this early age. A larger number of families with adult onset maternally inherited hearing impairment need to be studied to determine the frequency of the A $1555 \mathrm{G}$ mutation in the UK population.

Within our sample, mtDNA mutations were most common $(17.6 \%)$ in those with a family history of hearing impairment in more than one generation, the prevalence rising to $30 \%$ where affected subjects were related only through the maternal lineage. Our study also indicated the need for formal documentation of the cause of hearing impairment provided by the family history before making any assumptions about the mode of inheritance and screening for DNA mutations. The identification of an mtDNA mutation is vital for subsequent genetic counselling.

Further studies in other populations are required to determine the prevalence of mtDNA mutations in congenital/childhood NSSHI. Population differences are likely to exist although the A1555G, A3243G, and A7445G mutations have all been found on different ethnic backgrounds. The 7472insC mutation has also occurred multiple times in the white population ${ }^{13}$ indicating that routine screening for each of the mutations is worthwhile in congenital/childhood NSSHI, particularly where it is progressive and in any family where it might be maternally inherited. Other, as yet unidentified mtDNA mutations might also exist, accounting for further cases of NSSHI.

Work in the authors' laboratories is supported by the National Lottery Charities Board through Defeating Deafness, the West Riding Medical Research Trust, the Medical Research Council, European Community Framework V, and the Wellcome Trust.

1 Marazita ML, Ploughman LM, Rawlings B, Remington E, Arnos KS, Nance WE. Genetic epidemiological studies of early-onset deafness in the US school-age population. $A m \mathcal{F}$ Med Genet 1993;46:486-91.

2 Fischel-Ghodsian N. Mitochondrial deafness mutations reviewed. Hum Mutat 1999;13:261-70.

3 Hutchin TP, Cortopassi GA. Mitochondrial defects and hearing loss. Cell Mol Life Sci (in press).

4 Tiranti V, Chariot P, Carella F, Toscano A, Soliveri P, Girlanda P, Carrara F, Fratta GM, Reid FM, Mariotti C, Zeviani M. Maternally inherited hearing loss, ataxia and myoclonus associated with a novel point mutation in mitochondrial tRNASer(UCN) gene. Hum Mol Gemet 1995;4: 1421-7.

5 Hutchin TP, Parker MJ, Young ID, Davis AC, Pulleyn LJ, Deeble J, Lench NJ, Markham AF, Mueller RF. A novel mutation in the mitochondrial tRNA ${ }^{\text {ser(UCN) }}$ gene in a fammutation in the mitochondrial tRNA ${ }^{\mathrm{Ser}(\mathrm{UCN})}$ gene in a fam-
ily with maternally inherited hearing impairment. $f \mathrm{Med}$ Genet 2000;37:692-4.

6 Hutchin TP, Lench NJ, Arbuzova S, Markham AF and Mueller RF. Maternally inherited hearing impairment in a family with the mitochondrial DNA A7445G mutation. Eur f Hum Genet 2001;9:56-8.

7 Lehtonen MS, Uimonen S, Hassinen IE, Majamaa K. Frequency of mitochondrial DNA point mutations among patients with familial sensorineural hearing impairment. Eur F Hum Genet 2000;8:315-18.

8 Hutchin TP. Sensorineural hearing loss and the 1555G mitochondrial DNA mutation. Acta Otolaryngol (Stockh) 1998;118:48-52.

9 Estivill X, Govea N, Barcelo E, Badenas C, Romero E, Moral L, Scozzri R, D'Urbano L, Zeviani M, Torroni A. Familial progressive sensorineural deafness is mainly due to the mtDNA A $1555 \mathrm{G}$ mutation and is enhanced by treatment of aminoglycosides. Am 7 Hum Genet 1998;62: $27-35$.

10 Reid FM, Vernham GA, Jacobs HT. A novel mitochondrial point mutation in a maternal pedigree with sensorineural deafness. Hum Mutat 1994;3:243-7.

11 Jaksch M, Klopstock T, Kurlemann G, Dorner M, Hofmann S, Kleinle S, Hegemann S, Weissert M, Muller-Hocker J, Pongratz D, Gerbitz KD. Progressive myoclonus epilepsy and mitochondrial myopathy associated with mutations in the tRNA(Ser(UCN)) gene. Ann Neurol 1998;44:635-40.

12 Usami S, Abe S, Akita J, Namba A, Shinkawa H, Ishii M, Iwasaki S, Hoshino T, Ito J, Doi K, Kubo T, Nakagawa T, Komiyama S, Tono T, Komune S. Prevalence of mitochondrial gene mutations among hearing impaired patients. $\mathcal{f}$ Med Genet 2000;37:38-40.

13 Hutchin TP, Navarro-Coy NC, Van Camp G, Tiranti V, Zeviani M, Schuelke M, Jaksch M, Newton V, Mueller RF. Multiple origins of the mtDNA 7472ins $C$ mutation associated with hearing loss and neurological dysfunction. Eur $\mathcal{f}$ Hum Genet (in press). 